analysis of colonic macrophages was assessed as was the presence of goblet cells and mucosal T cells. In addition, we analyzed the development of goblet cell differentiation in colonoids in a co-culture system with proinflammatory macrophages. RESULTS/ANTICIPATED RESULTS: Our chronic inflammation models revealed an increase in proinflammatory macrophages present in the lamina propria and that these cells expressed significantly higher levels of notch ligand, Jagged1. Jagged 1 has been shown to enhance TH1 differentiation and $\mathrm{T}$ cells isolated from the mucosa of both chronic colitis models display strong TH1 skewing compared to controls. Chronic inflammation also contributes to intestinal barrier defects, enhanced permeability and bacterial translocation. We believe this enhanced intestinal permeability and subsequent bacterial translocation promote Jagged 1 expression in intestinal macrophages. To support this concept, we show TLR stimulation induces the upregulation of Jagged1 in BMM. Additionally, the generation of BMM from our chronic DSS-induced colitis mice or age matched controls, revealed BMM derived from a host of chronic inflammation were skewed to a proinflammatory state prior to stimulation showing increased gene expression of several proinflammatory molecules including IL- $1 \alpha$, IL- $1 \beta$, IL-12 and TNF- $\alpha$. This would suggest monocytes migrating to the intestinal mucosa have more potential to become proinflammatory instead of traditional anti-inflammatory macrophages. Furthermore, proinflammatory notch ligand-positive macrophages co-cultured with colonoids, derived from unperturbed mice, significantly decreased the number of mucus producing goblet cells. In support of this observation, notch activation in intestinal stem cells promote absorptive (i.e. colonocytes) cell differentiation and prevents secretory cell (i.e. goblet cells) differentiation. DISCUSSION/SIGNIFICANCE OF IMPACT: Taken together, our results strongly suggest chronic inflammation modulates macrophages role in maintaining intestinal homeostasis through possible notch activation in both $\mathrm{T}$ cells and the intestinal epithelial barrier.

3508

\section{Ciclopirox Olamine Demonstrates Inhibitory Effects on Esophageal Tumor Cells}

Randi Ryan ${ }^{1}$, Shrikant Anant ${ }^{2}$, Prabhu Ramamoorthy, PhD ${ }^{2}$, Dharmalingam Subramaniam, $\mathrm{PhD}^{2}$ and Scott Weir, PharmD, $\mathrm{PhD}^{2}$ ${ }^{1}$ University of Kansas Frontiers and ${ }^{2}$ University of Kansas Medical Center

OBJECTIVES/SPECIFIC AIMS: Drug repositioning has the potential to accelerate translation of novel cancer chemotherapeutics from bench to bedside. The goal of this study was to determine the effects of ciclopirox olamine (CPX) on esophageal tumor cells. METHODS/ STUDY POPULATION: We tested the effect of CPX on four esophageal cancer cell lines, assessing cell proliferation and viability by hexosaminidase and clonogenicity assay, respectively. We analyzed the effects of CPX on three-dimensional (3D) esophageal tumor cell spheroids. We also analyzed effects on cell cycle by flow cytometry. For mechanism, we performed western blots for proteins involved in cell cycle regulation, apoptosis and the $\mathrm{Wnt} / \beta$-catenin pathway. For in vivo effects, we performed a murine xenograft model with intraperitoneal administration of CPX (100 mg/Kg body weight daily). RESULTS/ANTICIPATED RESULTS: CPX inhibited growth of all cell lines in a time and concentration-dependent manner. CPX also inhibited growth of esophageal spheroids. Cell cycle analysis demonstrated G0/G1 arrest in cells treated with CPX. Western blot analyses demonstrated decreased expression of cyclinD1, CDK4, CDK6, and transcriptionally active $\beta$-catenin, supporting the role of CPX in cell cycle inhibition and decreased $\beta$-catenin activity. Finally, treatment of nude mice with CPX significantly decreased tumor xenograft volume. DISCUSSION/SIGNIFICANCE OF IMPACT: CPX demonstrates anti-tumor properties in esophageal cancer cell lines. The current results justify further research into the mechanism of this inhibition. Additionally, given its established safety in humans, CPX is a potential candidate for repositioning as an adjunct treatment for esophageal cancer.

Coagulation Factor Xll-mediated contact system and its role in adaptive and innate immune cell movement Emily Beltran ${ }^{1}$, Tukisa Smith ${ }^{1}$, Beth Graczyk ${ }^{1}$, Jan Breslow ${ }^{1}$ and Manish Ponda, M.D. ${ }^{1}$

${ }^{1}$ Rockefeller University

OBJECTIVES/SPECIFIC AIMS: The objectives of this study are to 1) expand upon the paradigm of HK-D5 accelerated immune cell chemotaxis; 2) characterize the role of FXII in murine models of immune-mediated disease using FXII KO mice and a small molecule inhibitor of FXIIa. METHODS/STUDY POPULATION: To test whether the addition of HK-D5 peptide would accelerate C-C chemokine receptor type 2 (CCR2)-mediated chemotaxis in vitro, a realtime transwell chemotaxis assay was developed utilizing human THP-1 monocyte cell line (Fig 1). For in vivo studies, both pharmacologic FXIIa antagonism and FXll KO mice were used. Genotyping, histopathological review, FXll protein expression, and active partial thromboplastin time (aPTT) measurements were used to phenotypically characterize FXII KO mice compared to C57BL/6 wild type mice (Fig 2). Subsequently, the FXll KO mice were studied in varied models of immune-mediated disease, including LPS-induced sepsis and experimental autoimmune encephalitis (EAE), to see if and how the absence of FXll can mitigate disease severity. The EAE model involved active immunization with myelin oligodendrocyte glycoprotein (MOG) and measurement of established clinical disease severity scores. The LPS sepsis model involved an intraperitoneal injection of LPS followed by 48-hour monitoring of core body temperature using subdermal temperature transponders as a proxy for inflammatory events related to septic shock (Fig 3). RESULTS/ ANTICIPATED RESULTS: HK-D5 significantly accelerates CCR2mediated chemotaxis compared to chemokine alone $(\mathrm{p}=0.001)$ similar to HK-D5's ability to accelerate CCR7-mediated chemotaxis as previously established. The FXIl KO mice were backcrossed to the C57BL/6J background and confirmed by genotyping and complete absence of FXII protein in plasma. Compared to the control, FXII KO mice have a significantly prolonged aPTT without evidence of bleeding abnormalities, which confirms the expected phenotype previously described and recapitulates what is observed in Factor XII deficiency in humans. KO mice showed no significant gross or histopathological differences in secondary lymphoid structures compared to the control. Immunohistochemistry confirmed wellorganized lymphoid structures with intact $\mathrm{B}$ - and T-cell populations. FXll KO mice are protected in LPS-induced septic shock and EAE models. Regarding the EAE model, FXIIa inhibition significantly reduced disease severity compared to control. In the LPS model, FXll KO mice recover within 24 hours after LPS-challenge measured subjectively and objectively by core body temperature measurement. DISCUSSION/SIGNIFICANCE OF IMPACT: The current study 